\title{
TARCO-Nomenklatur von Cockpit verabschiedet
}

\author{
Christian Oeschgera, Patrick Müller \\ ${ }^{a}$ Experte, Abteilung Ambulante Versorgung und Tarife; ${ }^{b}$ Abteilungsleiter Ambulante Versorgung und Tarife
}

Am 29. November 2017 hat bereits die sechste Sitzung des Steuerungsorgans des Projektes TARCO, genannt Cockpit, stattgefunden. Die Cockpit-Delegierten haben anlässlich dieser Sitzung die fast vollständige Nomenklatur der innerhalb der FMH revidierten Tarifstruktur "TARCO» in einer finalen Version verabschiedet. Dem Entscheid waren neun intensive Monate vorausgegangen, während deren die Experten der FMH in Zusammenarbeit mit allen der Ärzteverbindung angeschlossenen Fachgesellschaften die in der Urabstimmung 2016 abgelehnte Tarifstruktur überarbeitet hatten.

\begin{abstract}
Nachdem die abstimmenden Mitglieder der FMH im Juni 2016 den erarbeiteten Revisionsvorschlag für den ambulanten ärztlichen Tarif mehrheitlich abgelehnt hatten, erteilte die Delegiertenversammlung dem Zentralvorstand und dem Departement Ambulante Versorgung und Tarife im September 2016 ein neues Mandat zur Fortsetzung der Tarifrevision. Im Februar 2017 starteten die Arbeiten im Rahmen des Projektes TARCO (= TARMED Consensus) mit allen Fachgesellschaften.
\end{abstract}

Die FMH-Experten überarbeiteten die einzelnen Kapitel in über 30 verschiedenen Arbeitsgruppen mit den zuvor festgelegten und unter allen Fachgesellschaften vernehmlassten sogenannten «Leading-Fachgesellschaften». Die Ärztinnen und Ärzte revidierten mit den Tarifexperten die Kapitelstruktur, nahmen aber vor allem auch die einzelnen Tarifpositionen ein weiteres Mal unter die Lupe: In vielen Sitzungen und unzähligen E-Mails wurden Minutagen nochmals überarbeitet, medizinische Interpretationen ergänzt und umformuliert sowie die «Qualitativen Dignitäten» wieder eingeführt und auf alle Tarifpositionen festgelegt. Anders als im Vorgängerprojekt wurde anschliessend jedes Kapitel während sechs Wochen bei den anderen Fachgesellschaften vernehmlasst. Die 500 eingegangenen Feedbacks wurden an die zuständigen Arbeitsgruppen zurückgespiegelt. Dort wurden alle Rückmeldungen gesichtet, wo sinnvoll eingearbeitet und an die Fachgesellschaften zurückgemeldet, welche Feedbacks aus welchen Gründen keine Berücksichtigung fanden. Die Expertengruppe hat es mit diesem Vorgehen ge- schafft, auf breiter Basis und unter allen beteiligten Fachgesellschaften einen grösstmöglichen Konsens zu erreichen.

\section{Das Cockpit hat sich bewährt}

Wesentlich zum Gelingen hat auch die Organisationsstruktur beigetragen. Erstmals und anders als im Vorprojekt hat die FMH mit dem Cockpit ein unter allen FMH-Organisationen breit abgestütztes Steuerungsorgan eingesetzt. Im Cockpit sind folgende Organisationen vertreten:

- Verband Deutschschweizerischer Ärztegesellschaften (VEDAG, 1 Sitz),

- Société Médicale de la Suisse Romande (SMSR, 1 Sitz)

- Ordine dei Medici del Cantone Ticino (OMCT, 1 Sitz)

- Foederatio Medicorum Psychiatricorum et Psychotherapeuticorum (FMPP, 2 Sitze)

- Foederatio Medicorum Chirurgicorum Helvetica (FMCH, 2 Sitze)

- Kollegium für Hausarztmedizin (KHM, 2 Sitze)

- Swiss Federation of Specialities in Medicine (SFSM, 2 Sitze)

- Verein der Leitenden Spitalärzte der Schweiz (VLSS, 2 Sitze)

- Schweizerischer Belegärzte-Verband (SBV, 1 Sitz, Details siehe unten)

Ebenfalls eingeladen war der Verband der Schweizerischen Assistenz- und Oberärztinnen und -ärzte (VSAO, 2 Sitze). Anerkannte Fachgesellschaften (Anhang 2 der FMH-Statuten), die keinem Dachverband angeschlos- 


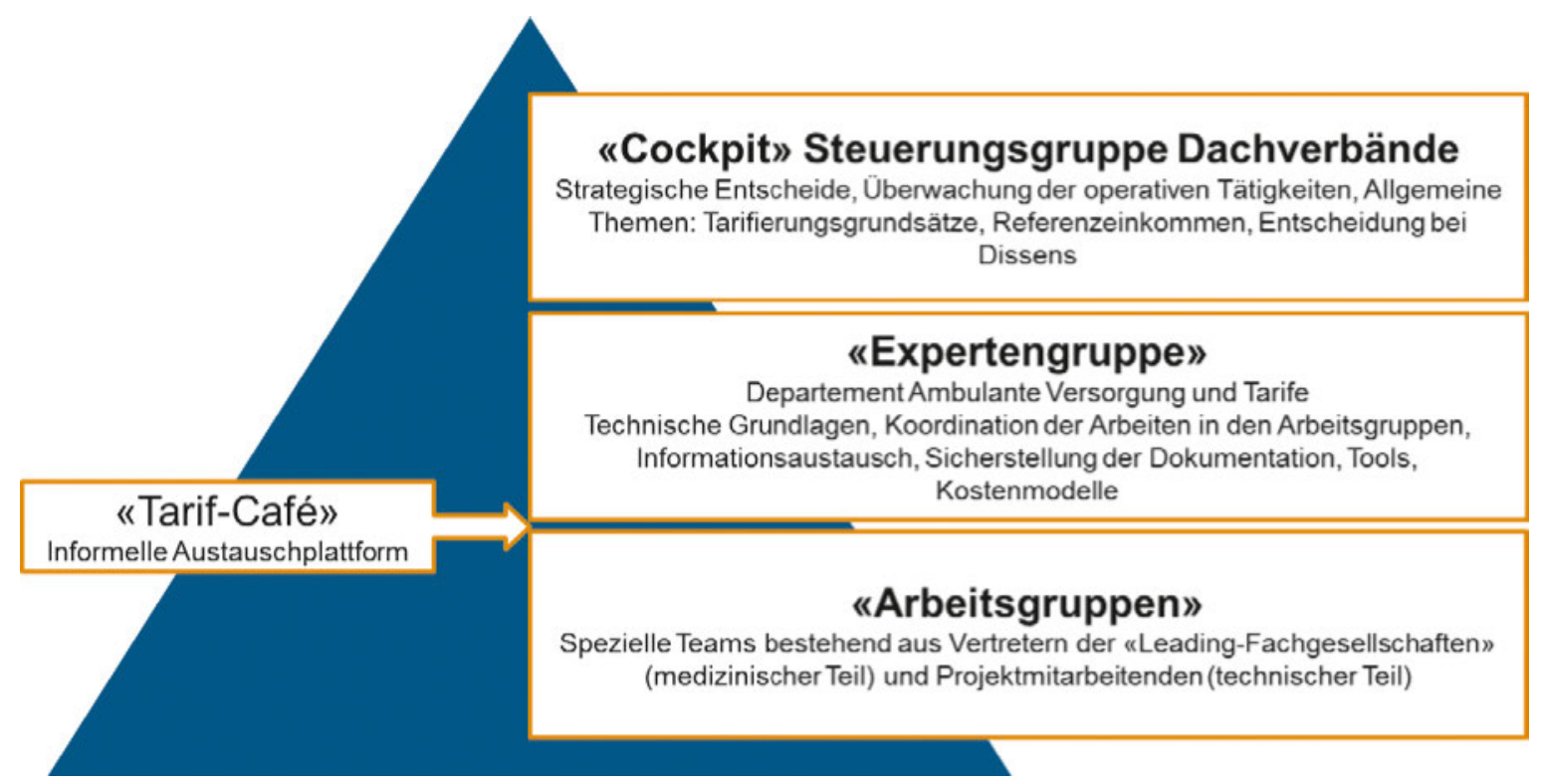

Projektorganisation TARCO.

sen sind sowie Organisationen, die gemäss den FMHStatuten mitspracheberechtigt sind (Anhang 3), werden durch den Schweizerischen Belegärzteverband (1 Sitz) repräsentiert. Die involvierten Gesellschaften hatten sich entsprechend darauf verständigt.

Die Cockpit-Delegierten hatten in den vergangenen Monaten in zahlreichen Sitzungen immer wieder über verschiedene Anträge seitens der Arbeitsgruppen bzw. der Fachgesellschaften zu beraten und trafen insgesamt über 100 Entscheidungen. Gegenstand der Entscheide waren Tarifierungsgrundsätze, die Nomenklatur oder die «Qualitativen Dignitäten».

\section{Finale Nomenklatur liegt vor}

In der sechsten Sitzung des Cockpits Ende November 2017 legte die Expertengruppe mit Ausnahme von zwei Kapiteln («Bewegungsapparat und Handchirurgie» und "Allgemeine Leistungen im Operationssaal») alle Tarifpositionen den Delegierten zur Genehmigung vor. Gegenüber der Version, die in der Urabstimmung scheiterte, wurden zahlreiche Kapitel erneut überarbeitet. So ist die Gesamtzahl der Positionen nochmals etwas angestiegen (2700 Positionen), liegt aber immer noch signifikant tiefer als im heute gültigen TARMED (aktuell rund 4500 Positionen). Des Weiteren gibt es neu unter anderen auch spezifische Tarifpositionen in den Bereichen "Medizinische Genetik», "Altersmedizin», «Hyperbarmedizin», «Infektiologie», «Nephrologie» und «Medizinische Onkologie und Hämatologie». Das Cockpit hat sämtliche vorgelegten Kapitel verabschiedet und der Expertengruppe der FMH den Auftrag erteilt, die Tarifstruktur mit den Tarifpartnern zu verhandeln. Die beiden noch ausstehenden Kapitel werden voraussichtlich in der Cockpit-Sitzung vom 10. Januar 2018 verabschiedet und werden dann ebenfalls in die Verhandlungen einfliessen.

\section{Verhandlungen mit Tarifpartnern laufen bereits}

Innerhalb der ats-tms AG sind die Gespräche auf der Ebene der Kostenmodelle bereits gestartet. Die FMH bzw. ihre Datenlieferanten zur Berechnung der Kostensätze - die Ärztekasse und die kantonalen Ärztegesellschaften sowie die NewIndex AG - sind aufgefordert, beim Kostenmodell KOREG grössere Transparenz herzustellen. Um diese Transparenz gewährleisten zu können, laufen aktuell intensive Gespräche, da die Daten für die FMH und ihre Mitglieder von hoher Wichtigkeit sind. Entscheidend ist, dass die FMH keine Einzeldaten liefern wird und die aggregierten Daten nur für die Geschäftsstelle der ats-tms AG, nicht aber für die einzelnen Gesellschafter/Tarifpartner einsehbar sind. Zudem soll eine Prüfung der Rohdaten nur über eine unabhängige Treuhandstelle möglich sein.

Aufgrund zahlreicher Rückmeldungen von Fachgesellschaften hat die FMH die Tarifpartner $\mathrm{H}+$, curafutura und MTK dazu aufgefordert, auch das Kostenmodell INFRA nochmals zu überprüfen. Die Experten haben sich entschieden, nicht alle Sparten-Parameter nochmals zu kontrollieren und dies auch nicht bei allen INFRA-Sparten. Überprüft werden nochmals alle Sparten, über die entweder ein grosses Taxpunktvolumen 
Korrespondenz: FMH / Abteilung Ambulante Versorgung und Tarife Baslerstrasse 47 $\mathrm{CH}-4600$ Olten

Tel. 0313591230 Fax 0313591238 tarife.ambulant[at]fmh.ch generiert wird oder deren Personal- oder Anlagenutzungskosten sehr hoch sind. Zudem werden auch Sparten nochmals angeschaut, die - verglichen mit den Kostensätzen der TARMED-Version 01.08 .00 - eine überproportionale Kostensteigerung aufweisen. Werden die Parameter einer Sparte nochmals überprüft, so werden aus Gründen der ganzheitlichen Betrachtung auch die "Nachbarsparten" erneut unter die Lupe genommen.

\section{Weiterer Zeitplan}

In den kommenden Wochen wird die FMH neben den Kostenmodellen auch die Nomenklatur mit den Tarifpartnern curafutura, H+ und MTK verhandeln. Dazu sind die Experten der FMH bereits in Kontakt mit der ats-tms AG. Innerhalb der gemeinsamen Organisation wird schon seit längerem über die zentralen Positionen aus dem Bereich «ärztliche und nichtärztliche Grundleistungen" sowie über übergreifende Themen wie die Berichts- und Befundungspositionen verhandelt. Des Weiteren sind Ende letztes Jahr auch die Nomenklatur-Verhandlungen aufgenommen worden.
Die FMH wird zusammen mit den ärztlichen Experten aus den Fachgesellschaften die TARCO-Nomenklatur in die Verhandlungen miteinbringen. Gleichzeitig wird die Kostenträgerseite ihre Forderungen einbringen (z.B. Limitationen).

Die FMH hält am Ziel fest, die revidierte Struktur zusammen mit den Tarifpartnern per 30. Juni 2018 beim Bundesrat zur Genehmigung einzureichen. Zuerst muss die Struktur nach erfolgreichen Verhandlungen, im Frühjahr 2018 aber sowohl von der Delegiertenversammlung der FMH sowie anschliessend von der Ärztekammer genehmigt werden.

\section{TARCO als einzige Alternative zum Amtstarif}

Für die FMH ist eine umfassende Gesamtrevision des TARMED, gemeinsam mit allen Ärzteorganisationen und Tarifpartnern, der einzig richtige Weg. Nach dem Tarifeingriff des Bundesrates ist es umso wichtiger, jetzt endlich eine sachgerechte und betriebswirtschaftliche Tarifstruktur in Kraft zu setzen. 